\title{
Bulk Arrival Two Phase Retrial Queueing System with Impatient Customers, Orbital Search, Active Breakdowns and Delayed Repair
}

\author{
J.Ebenesar Anna Bagyam \\ Assistant Professor in Mathematics \\ SNS College of Technology \\ Coimbatore
}

\author{
K.Udaya Chandrika,Ph.D \\ Professor in Mathematics \\ Avinashilingam Deemed University \\ for Women, Coimbatore
}

\author{
K.Prakash Rani,Ph.D \\ Associate Professor \\ ADM College for Women \\ Nagapattinam
}

\begin{abstract}
Bulk arrival general service retrial queueing system is analyzed in this paper. Server provides two phases of serviceessential and optimal. After each service completion, the server searches for customers in the orbit. Customers may balk or renege at particular times. Accidental and active breakdown of the server is considered. The repair of the failed server starts after a random amount of time known as delay time. After repair the server continues the service of the interrupted customer or waits for the same customer. The necessary and sufficient condition for the system to be stable is presented. By applying supplementary variable technique, the steady state distributions of the server state and the number of customers in the orbit are obtained. Numerical examples are presented to illustrate the influence of the parameters on several performance characteristics
\end{abstract}

Key Words - Retrial Queue, Server Breakdown, Orbital Search, Delayed Repair, Reserved Time.

\section{INTRODUCTION}

During the last two decades considerable attention has been paid to the analysis of queueing system with repeated calls see for example the book by Falin and Templeton [10], Artalejo and Gomez-Corral [2] and the survey papers of Artalejo [3, 4].

In many waiting line systems, the role of the server is played by mechanical / electronic device, such as computer, ATM, traffic light etc., which is subject to accidental random failure; until the failed server is repaired, it may cause a halt in service. Wang [17], Wang and Li [18], Atencia et. al [6], Geni Gupur [11] and Aissani [1] have studied retrial queueing system with random breakdown. In most of the articles with server breakdown it is assumed that the failed service channel undergoes repair instantaneously. But in several life situations, men have to be employed who may not be available on call to affect the necessary repair. Consequently a delay is caused in starting the repair of the breakdown server. Only few authors, for example Prakash Rani et.al $[12,13]$ and Rehab et. al [14] have analysed unreliable queueing models with delayed repair.

The next specific feature considered in retrial queueing system is orbital search. Artalejo et al. [5], Dudin et al. [8], Chakravarthy et al. [7] and Udaya Chandrika and Ebenesar Annabagyam [15] have investigated retrial queue with orbital search to reduce the idle time of the server.
In this paper, bulk arrival two phase retrial queueing system with balking, reneging, orbital search and server breakdown is discussed by including the concept of delay time and reserved time. Analytical treatment of this model is obtained by supplimentry variable technique. The main motivation is from the applications to Local Area Networks, client server communication and electronic mail services on internet.

\section{MODEL DESCRIPTION}

Consider a single server retrial queue in which customers arrive in batches of variable size according to a Poisson process with rate $\lambda$. The batch size $\mathrm{Y}$ is a random variable with probability mass function $\mathrm{P}(\mathrm{Y}=\mathrm{k})=\mathrm{C}_{\mathrm{k}}$, the probability generating function $\mathrm{C}(\mathrm{z})$ and first two moments $\mathrm{m}_{1}$ and $\mathrm{m}_{2}$. At the arrival epoch of a batch, if the server is idle, then one of the customers get into the service immediately and others leave the service area and enter the retrial queue. If the server is busy or down, the arriving customers either join a retrial queue with probability $\mathrm{p}$ or leave the system with probability $1-\mathrm{p}$. The customer at the head of the retrial queue competes with potential primary customers to decide which customer will enter service next. If a primary customer arrives first, the retrial customer may cancel its attempt for service and either return to its position in the retrial queue with probability $\mathrm{q}$ or quit the system with probability $1-\mathrm{q}$. The retrial time of the customer in the retrial queue is generally distributed with distribution function $\mathrm{A}(\mathrm{x})$, Laplace Stieltjes transform $A^{*}(s)$ and the hazard rate function $\eta(\mathrm{x})$.

The server provides two phases of service phase 1 and phase 2. The phase 1 service is needed to all arriving customers. As soon as the phase 1 service is completed, the customer may opt to leave the system probability $\tau$ or opt for the phase 2 service with probability $1-\tau$.

The service times of phase $\mathrm{i}$ are generally distributed with distribution function $\mathrm{B}_{\mathrm{i}}(\mathrm{x})$, Laplace Stieltjes transform $\mathrm{B}_{\mathrm{i}}^{*}(\mathrm{~s})$, the hazard rate function $\mu_{\mathrm{i}}(\mathrm{x})$ and the first two moments $\mu_{\mathrm{i} 1}$ and $\mu_{\mathrm{i} 2}$. If the server becomes free after completing phase $\mathrm{i}$ service, then immediately the server searches for customers in the orbit (if any) with probability $1-\rho_{\mathrm{i}}$ or remains idle with probability $\rho_{\mathrm{i}}$.

Assume that the server is subject to fail while it is busy in any one of the two phases. The life time of the server in $i^{\text {th }}$ phase is exponentially distribution with mean $\alpha_{\mathrm{i}}$. When the server breaks down, there is some delay to start the repair. The delay 
time distributions in both the phases are arbitrarily distributed with probability distribution functions $\mathrm{V}_{\mathrm{i}}(\mathrm{x})$, Laplace Stieltjes transform $v_{i}^{*}(s)$, the hazard rate function $\gamma_{i}(x)$ and the first two moments $v_{i 1}, v_{i 2}$. The repair time in both service phases are also generally distributed with probability distribution functions $\mathrm{H}_{\mathrm{i}}(\mathrm{x})$, Laplace Stieltjes transform $\mathrm{H}_{\mathrm{i}}^{*}(\mathrm{~s})$, the hazard rate function $\beta_{\mathrm{i}}(\mathrm{x})$ and the first two moments $h_{i 1}, h_{i 2}$.

When the server fails in phase $\mathrm{i}$ service, the customer in service either remains in the service position with probability $r$ until the server is up or leaves the service area with probability 1 $-r_{i}$ and keeps returning at times exponentially distributed with the rate $\theta_{\mathrm{i}}$. If the customer in service leaves the service area upon server failure, the server after repair completion, waits for the same customer to return. This time is referred as reserved time. The server is not allowed to accept new customers until the interrupted customer leaves the system after completion of service. The server is said to be blocked if the server is busy or down.

At time $\mathrm{t}$, let $\mathrm{N}(\mathrm{t})$ be the number of customers in the retrial queue, $\mathrm{W}(\mathrm{t})$ the elapsed retrial time of the customer, $\mathrm{X}(\mathrm{t})$ the elapsed service time of the customer in service, $\mathrm{U}(\mathrm{t})$ the elapsed delay time in attending the failed server, $\mathrm{Y}(\mathrm{t})$ the elapsed repair time of the server and $\mathrm{R}(\mathrm{t})$ the elapsed reserved time.

Define the following state probabilities:

$\mathrm{I}_{0}(\mathrm{t})$ is the probability that the server is idle at time $\mathrm{t}$ and there is no customer in the retrial orbit.

$I_{n}(t, w) d w$ is the joint probability that at time $t$ there are $n$ customers in the orbit, the server is idle and the elapsed retrial time of an orbital customer is between $w$ and $w+d w, n \geq 1$.

$P_{n}^{i}(t, x) d x$ is the joint probability that at time $t$ there are $n$ customers in the retrial orbit, the server is providing phase $i$ service with the elapsed service time between $\mathrm{x}$ and $\mathrm{x}+\mathrm{dx}$, where $\mathrm{n} \geq 0$.

$D_{0, n}^{i}(t, x, u) d x d u\left(D_{1, n}^{i}(t, x, u) d x d u\right) \quad$ is the joint probability that at time $t$ there are $n$ customers in the retrial orbit, the server is down, the elapsed service time is $\mathrm{x}$, the interrupted customer remains in the service position (not in the service position) and the elapsed delay time is between $u$ and $u+d u$, where $\mathrm{n} \geq 0$.

$$
\mathrm{F}_{0, \mathrm{n}}^{\mathrm{i}}(\mathrm{t}, \mathrm{x}, \mathrm{y}) \mathrm{dx} \text { dy }\left(\mathrm{F}_{1, \mathrm{n}}^{\mathrm{i}}(\mathrm{t}, \mathrm{x}, \mathrm{y}) \mathrm{dx}\right. \text { dy) is the joint }
$$
probability that at time $t$ there are $n$ customers in the retrial orbit, the server is down, the elapsed service time is $\mathrm{x}$, the interrupted customer remains in the service position (not in the service position) and the elapsed repair time is between $\mathrm{y}$ and $\mathrm{y}+\mathrm{dy}$, where $\mathrm{n} \geq 0$.

$\mathrm{R}_{\mathrm{n}}^{\mathrm{i}}(\mathrm{t}, \mathrm{x}, \mathrm{r}) \mathrm{dx} \mathrm{dr}$ is the joint probability that at time $\mathrm{t}$ there are $\mathrm{n}$ customers in the retrial orbit, the elapsed service time is equal to $\mathrm{x}$ and the elapsed reserve time is between $\mathrm{r}$ and $\mathrm{r}+\mathrm{dr}$, where $\mathrm{n} \geq 0$.

\subsection{Theorem}

The necessary and sufficient condition for the system is to be stable is

$\lambda \operatorname{pm}_{1}\left[\mu_{11}\left(1+\alpha_{1}\left(\frac{1-\mathrm{r}_{1}}{\theta_{1}}+\mathrm{h}_{11}+\mathrm{v}_{11}\right)\right)+(1-\tau) \mu_{21}\left(1+\alpha_{2}\left(\frac{1-\mathrm{r}_{2}}{\theta_{2}}+\right.\right.\right.$ $\left.\left.\left.\mathrm{h}_{21}+\mathrm{v}_{21}\right)\right)\right]<1-\left(1-\mathrm{A}^{*}(\lambda)\right)\left(\mathrm{q}+\mathrm{m}_{1}-1\right)\left(\tau \rho_{1}+\rho_{2}(1-\tau)\right)$

Proof

Let $S^{(\mathrm{k})}$ be the generalized service time of the $\mathrm{k}^{\text {th }}$ customer in service. Then $\left\{S^{(\mathrm{k})}\right\}$ are independently and identically distributed with Laplace transform

$$
\begin{aligned}
\mathrm{B} *(\mathrm{~s}) & =\mathrm{B}_{1} *\left(\mathrm{~s}+\alpha_{1}-\alpha_{1}\left(\frac{\mathrm{r}_{1} \mathrm{~s}+\theta_{1}}{\mathrm{~s}+\theta_{1}} \mathrm{H}_{1} *(\mathrm{~s}) \mathrm{V}_{1} *(\mathrm{~s})\right)\right) \\
& +(1-\tau) \mathrm{B}_{2} *\left(\mathrm{~s}+\alpha_{2}-\alpha_{2}\left(\frac{\mathrm{r}_{2} \mathrm{~s}+\theta_{2}}{\mathrm{~s}+\theta_{2}} \mathrm{H}_{2} *(\mathrm{~s}) \mathrm{V}_{2} *(\mathrm{~s})\right)\right)
\end{aligned}
$$

and expected value

$$
\begin{aligned}
\mathrm{E}\left(\mathrm{S}^{(\mathrm{k})}\right) & =\mu_{11}\left(1+\alpha_{1}\left(\frac{1-\mathrm{r}_{1}}{\theta_{1}}+\mathrm{h}_{11}+\mathrm{v}_{11}\right)\right) \\
& +(1-\tau) \mu_{21}\left(1+\alpha_{2}\left(\frac{1-\mathrm{r}_{2}}{\theta_{2}}+\mathrm{h}_{21}+\mathrm{v}_{21}\right)\right)
\end{aligned}
$$

Let $\mathrm{P}(\mathrm{S})$ and $\mathrm{P}(\mathrm{I})$ denote respectively the probabilities that the system is blocked and idle. Let $\mathrm{E}\left(\mathrm{S}^{(\mathrm{k})}\right)$ be the expected blocked time and $\mathrm{E}(\mathrm{I})$ be the expected idle time. Then

$$
\mathrm{P}(\mathrm{S})=\frac{\mathrm{E}\left(\mathrm{S}^{(\mathrm{k})}\right)}{\mathrm{E}\left(\mathrm{S}^{(\mathrm{k})}\right)+\mathrm{E}(\mathrm{I})} \text { and } \mathrm{P}(\mathrm{I})=\frac{\mathrm{E}(\mathrm{I})}{\mathrm{E}\left(\mathrm{S}^{(\mathrm{k})}\right)+\mathrm{E}(\mathrm{I})}
$$

The arrival rate at the retrial queue when the system is blocked is $\lambda \operatorname{pm}_{1} \mathrm{P}(\mathrm{S})$

The arrival rate at the retrial queue when the server is idle is $\left(1-\mathrm{A}^{*}(\lambda)\right)\left(\mathrm{m}_{1}-1\right)\left(\rho_{1} \tau+(1-\tau) \rho_{2}\right) \mathrm{P}(\mathrm{I}) / \mathrm{E}(\mathrm{I})$.

Total arrival rate at the retrial queue is $\lambda \mathrm{pm}_{1} \mathrm{P}(\mathrm{S})+$ $\left(1-\mathrm{A}^{*}(\lambda)\right)\left(\mathrm{m}_{1}-1\right)\left(\rho_{1} \tau+(1-\tau) \rho_{2}\right) \mathrm{P}(\mathrm{I}) / \mathrm{E}(\mathrm{I})$.

The exit rate from the retrial queue by entering service when there is no orbital search is $\left.A^{*}(\lambda)\right)\left(\rho_{1} \tau+(1-\tau) \rho_{2}\right) P(I) / E(I)$.

The exit rate from the retrial queue by the orbital search is $\left(\tau\left(1-\rho_{1}\right)+(1-\tau)\left(1-\rho_{2}\right)\right) P(I) / E(I)$.

The exit rate from the retrial queue by leaving the system when a primary customer arrives first at the server is $(1-q)\left(1-A^{*}(\lambda)\right)\left(\rho_{1} \tau+(1-\tau) \rho_{2}\right) P(I) / E(I)$.

The total exit rate from the retrial queue is $\left[1-\mathrm{q}\left(1-\mathrm{A}^{*}(\lambda)\right)\right.$ $\left.\left(\rho_{1} \tau+(1-\tau) \rho_{2}\right)\right] \mathrm{P}(\mathrm{I}) / \mathrm{E}(\mathrm{I})$

For stability, the arrival rate should be less than the exit rate. Hence $\lambda \operatorname{pm}_{1} \mathrm{P}(\mathrm{S})+\left(1-\mathrm{A}^{*}(\lambda)\right)\left(\mathrm{m}_{1}-1\right)\left(\rho_{1} \tau+(1-\tau) \rho_{2}\right) \mathrm{P}(\mathrm{I}) / \mathrm{E}(\mathrm{I})<$ $\left[1-\mathrm{q}\left(1-\mathrm{A}^{*}(\lambda)\right)\left(\rho_{1} \tau+(1-\tau) \rho_{2}\right)\right] \mathrm{P}(\mathrm{I}) / \mathrm{E}(\mathrm{I})$.

and hence

$\lambda \operatorname{pm}_{1}\left[\mu_{11}\left(1+\alpha_{1}\left(\frac{1-\mathrm{r}_{1}}{\theta_{1}}+\mathrm{h}_{11}+\mathrm{v}_{11}\right)\right)+(1-\tau) \mu_{21}\left(1+\alpha_{2}\left(\frac{1-\mathrm{r}_{2}}{\theta_{2}}+\mathrm{h}_{21}\right.\right.\right.$ $\left.\left.\left.+\mathrm{v}_{21}\right)\right)\right]<1-\left(1-\mathrm{A}^{*}(\lambda)\right)\left(\mathrm{q}+\mathrm{m}_{1}-1\right)\left(\rho_{1} \tau+(1-\tau) \rho_{2}\right)$. 


\section{STEADY STATE DISTRIBUTIONS}

The system of equations that governs the model under steady state, by supplementary variable method are

$\lambda \mathrm{I}_{0}=\tau \int_{0}^{\infty} \mathrm{p}_{0}^{(1)} \mu_{1}(\mathrm{x}) \mathrm{dx}+\int_{0}^{\infty} \mathrm{p}_{0}^{(2)} \mu_{2}(\mathrm{x}) \mathrm{dx}$

$\frac{\mathrm{dI}_{\mathrm{n}}(\mathrm{w})}{\mathrm{dw}}=-(\lambda+\eta(\mathrm{w})) \mathrm{I}_{\mathrm{n}}(\mathrm{w}), \mathrm{n} \geq 1$

$\frac{\mathrm{dP}_{\mathrm{n}}^{(\mathrm{i})}(\mathrm{x})}{\mathrm{dx}}=-\left(\mathrm{p} \lambda+\mu_{\mathrm{i}}(\mathrm{x})+\alpha_{\mathrm{i}}\right) \mathrm{P}_{\mathrm{n}}^{(\mathrm{i})}(\mathrm{x})+\int_{0}^{\infty} \mathrm{F}_{0, \mathrm{n}}^{(\mathrm{i})}(\mathrm{x}, \mathrm{y}) \beta_{\mathrm{i}}(\mathrm{y}) \mathrm{dy}$ $+\theta_{\mathrm{i}} \int_{0}^{\infty} \mathrm{R}_{\mathrm{n}}^{(\mathrm{i})}(\mathrm{x}, \mathrm{r}) \mathrm{dr}+\mathrm{p} \lambda\left(1-\delta_{0 \mathrm{n}}\right) \sum_{\mathrm{k}=1}^{\mathrm{n}} \mathrm{c}_{\mathrm{k}} \mathrm{P}_{\mathrm{n}-\mathrm{k}}^{(\mathrm{i})}(\mathrm{x})$,

$$
\mathrm{n} \geq 0, \mathrm{i}=1,2
$$

$\frac{\partial \mathrm{D}_{\mathrm{i}, \mathrm{n}}^{(\mathrm{i})}(\mathrm{x}, \mathrm{u})}{\mathrm{du}}=-\left(\mathrm{p} \lambda+\gamma_{\mathrm{i}}(\mathrm{u})\right) \mathrm{D}_{\mathrm{i}, \mathrm{n}}^{(\mathrm{i})}(\mathrm{x}, \mathrm{u})+\mathrm{p} \lambda\left(1-\delta_{0 \mathrm{n}}\right) \sum_{\mathrm{k}=1}^{\mathrm{n}} \mathrm{c}_{\mathrm{k}} \mathrm{D}_{\mathrm{i}, \mathrm{n}}^{(\mathrm{i})}(\mathrm{x}, \mathrm{u})$, $\mathrm{n} \geq 0, \mathrm{i}=1,2 ; \mathrm{j}=0,1$

$\frac{\partial \mathrm{F}_{\mathrm{j}, \mathrm{n}}^{(\mathrm{i})}(\mathrm{x}, \mathrm{y})}{\mathrm{dy}}=-\left(\mathrm{p} \lambda+\beta_{\mathrm{i}}(\mathrm{u})\right) \mathrm{F}_{\mathrm{j}, \mathrm{n}}^{(\mathrm{i})}(\mathrm{x}, \mathrm{y})+\mathrm{p} \lambda\left(1-\delta_{0 \mathrm{n}}\right) \sum_{\mathrm{k}=1}^{\mathrm{n}} \mathrm{c}_{\mathrm{k}} \mathrm{F}_{\mathrm{j}, \mathrm{n}}^{(\mathrm{i})}(\mathrm{x}, \mathrm{y})$,

$$
\mathrm{n} \geq 0, \mathrm{i}=1,2 ; \mathrm{j}=0,1
$$

$\frac{\partial \mathrm{R}_{\mathrm{n}}^{(\mathrm{i})}(\mathrm{x}, \mathrm{r})}{\mathrm{dr}}=-\left(\mathrm{p} \lambda+\theta_{\mathrm{i}}\right) \mathrm{R}_{\mathrm{n}}^{(\mathrm{i})}(\mathrm{x}, \mathrm{r})+\mathrm{p} \lambda\left(1-\delta_{0 \mathrm{n}}\right) \sum_{\mathrm{k}=1}^{\mathrm{n}} \mathrm{c}_{\mathrm{k}} \mathrm{R}_{\mathrm{n}}^{(\mathrm{i})}(\mathrm{x}, \mathrm{r})$,

$$
\mathrm{n} \geq 0, \mathrm{i}=1,2
$$

with boundary conditions

$I_{n}(0)=\tau \rho_{1} \int_{0}^{\infty} p_{n}^{(1)}(x) \mu_{1}(x) d x+\rho_{2} \int_{0}^{\infty} p_{n}^{(2)}(x) \mu_{1}(x) d x, n \geq 1$

$\mathrm{P}_{\mathrm{n}}^{(1)}(0)=\tau\left(1-\rho_{1}\right) \int_{0}^{\infty} \mathrm{p}_{\mathrm{n}+1}^{(1)}(\mathrm{x}) \mu_{1}(\mathrm{x}) \mathrm{dx}+\left(1-\rho_{2}\right) \int_{0}^{\infty} \mathrm{p}_{\mathrm{n}+1}^{(2)}(\mathrm{x}) \mu_{2}(\mathrm{x}) \mathrm{dx}$

$$
+\lambda \mathrm{C}_{\mathrm{n}+1} \mathrm{I}_{0}+\lambda(1-\mathrm{q}) \sum_{\mathrm{k}=1}^{\mathrm{n}+1} \mathrm{C}_{\mathrm{k}} \int_{0}^{\infty} \mathrm{I}_{\mathrm{n}-\mathrm{k}+2}(\mathrm{w}) \mathrm{dw}
$$

$+\int_{0}^{\infty} I_{n+1}(w) \eta(w) d w+\lambda q\left(1-\delta_{0 n}\right) \sum_{k=1}^{n} C_{k} \int_{0}^{\infty} I_{n-k+1}(w) d w$,

$$
\mathrm{n} \geq 1
$$

$P_{n}^{(2)}(0)=(1-\tau) \int_{0}^{\infty} p_{n}^{(1)}(x) \mu_{1}(x) d x, \quad n \geq 0$

$\mathrm{D}_{0, \mathrm{n}}^{(\mathrm{i})}(\mathrm{x}, 0)=\mathrm{r}_{\mathrm{i}} \alpha_{\mathrm{i}} \mathrm{P}_{\mathrm{n}}^{(\mathrm{i})}(\mathrm{x}), \mathrm{n} \geq 0, \mathrm{i}=1,2$

$\mathrm{D}_{1, \mathrm{n}}^{(\mathrm{i})}(\mathrm{x}, 0)=\left(1-\mathrm{r}_{\mathrm{i}}\right) \alpha_{\mathrm{i}} \mathrm{P}_{\mathrm{n}}^{(\mathrm{i})}(\mathrm{x}), \mathrm{n} \geq 0, \mathrm{i}=1,2$

$\mathrm{F}_{\mathrm{o}, \mathrm{n}}^{(\mathrm{i})}(\mathrm{x}, 0)=\int_{0}^{\infty} \mathrm{D}_{\mathrm{o}, \mathrm{n}}^{(\mathrm{i})}(\mathrm{x}, \mathrm{u}) \gamma_{\mathrm{i}}(\mathrm{u}) \mathrm{du}, \mathrm{n} \geq 0, \mathrm{i}=1,2$

$F_{1, n}^{(i)}(x, 0)=\int_{0}^{\infty} D_{1, n}^{(i)}(x, u) \gamma_{i}(u) d u, n \geq 0, i=1,2$

$\mathrm{R}_{\mathrm{n}}^{(\mathrm{i})}(\mathrm{x}, 0)=\int_{0}^{\infty} \mathrm{F}_{\mathrm{i}, \mathrm{n}}^{(\mathrm{i})}(\mathrm{x}, \mathrm{y}) \beta_{\mathrm{i}}(\mathrm{y}) \mathrm{dy}, \mathrm{n} \geq 0, \mathrm{i}=1,2$

Define the following probability generating functions $I(z, w)=\sum_{n=1}^{\infty} I_{0}(w) z^{n}, \quad P^{i}(z, x)=\sum_{n=0}^{\infty} P_{n}^{i}(x) z^{n}$,

$D_{j}^{i}(z, x, u)=\sum_{n=0}^{\infty} D_{j, n}^{i}(x, u) z^{n}, \quad F_{j}^{i}(z, x, y)=\sum_{n=0}^{\infty} F_{j, n}^{i}(x, y) z^{n}$,

$R^{i}(z, x, r)=\sum_{n=0}^{\infty} R_{n}^{i}(x, r) z^{n}, i=1,2 ; j=0,1$ and $|z| \leq \mid$

Proceeding in the usual manner with the equations (1)-(14) we can determine the expressions of $\mathrm{I}(\mathrm{z}, \mathrm{w}), \mathrm{P}^{(\mathrm{i})}(\mathrm{z}, \mathrm{x})$, $D_{j}^{(i)}(z, x, u), F_{j}^{(i)}(z, x, y)$ and $R^{(i)}(z, x, r), i=1,2 ; j=1,2$.
By defining the the partial probability generating function $\psi(\mathrm{z})=\int_{0}^{\infty} \psi(\mathrm{z}, \mathrm{x}) \mathrm{dx}$ or $\psi(\mathrm{z})=\int_{0}^{\infty} \int_{0}^{\infty} \psi(\mathrm{z}, \mathrm{x}, \mathrm{y}) \mathrm{dx} \mathrm{dy}$, we can find the queue size probability generating functions $\mathrm{I}(\mathrm{z}), \mathrm{P}^{(\mathrm{i})}(\mathrm{z})$, $\mathrm{D}_{\mathrm{j}}^{(\mathrm{i})}(\mathrm{z}), \mathrm{F}_{\mathrm{j}}^{(\mathrm{i})}(\mathrm{z})$ and $\mathrm{R}^{(\mathrm{i})}(\mathrm{z})$.

\section{PERFORMANCE MEASURES}

Taking $\mathrm{z}=1$ in $\mathrm{I}(\mathrm{z}), \mathrm{P}^{(\mathrm{i})}(\mathrm{z}), \mathrm{D}_{\mathrm{j}}^{(\mathrm{i})}(\mathrm{z}), \mathrm{F}_{\mathrm{j}}^{(\mathrm{i})}(\mathrm{z})$ and $\mathrm{R}^{(\mathrm{i})}(\mathrm{z})$ we obtain the following system state probabilities.

The steady state probability that the system is non-empty and the server is idle is $\mathrm{I}=\mathrm{I}(1)=\mathrm{I}_{0}\left(1-\mathrm{A}^{*}(\lambda)\right)\left[\mathrm{K}_{1}^{\prime}(1)+(1-\tau)\right.$ $\left.\mathrm{K}_{2}^{\prime}(1)+\mathrm{m}_{1}\left(\tau \rho_{1}+(1-\tau) \rho_{2}\right)-1\right] / \mathrm{T}_{1}$

The steady state probability that the server is busy is

$$
\begin{aligned}
\mathrm{P} & =\mathrm{P}^{(1)}(1)+\mathrm{P}^{(2)}(1) \\
& =\mathrm{I}_{0} \lambda \mathrm{N}_{2}\left[\mu_{11}+(1-\tau) \mu_{21}\right] / \mathrm{T}_{1}
\end{aligned}
$$

The steady state probability that the breakdown server waits for its repair to start is given by

$$
\begin{aligned}
\mathrm{D} & =\mathrm{D}_{0}^{(1)}(1)+\mathrm{D}_{1}^{(1)}(1)+\mathrm{D}_{0}^{(2)}(1)+\mathrm{D}_{0}^{(2)}(1) \\
& =\mathrm{I}_{0} \lambda \mathrm{N}_{2}\left[\alpha_{1} \mu_{11} \mathrm{v}_{11}+(1-\tau) \alpha_{2} \mu_{21} \mathrm{v}_{21}\right] / \mathrm{T}_{1}
\end{aligned}
$$

The steady state probability that the server is under repair is

$$
\begin{aligned}
\mathrm{F} & =\mathrm{F}_{0}^{(1)}(1)+\mathrm{F}_{1}^{(1)}(1)+\mathrm{F}_{0}^{(2)}(1)+\mathrm{F}_{0}^{(2)}(1) \\
& =\mathrm{I}_{0} \lambda \mathrm{N}_{2}\left[\alpha_{1} \mu_{11} \mathrm{~h}_{11}+(1-\tau) \alpha_{2} \mu_{21} \mathrm{~h}_{21}\right] / \mathrm{T}
\end{aligned}
$$
time is

The steady state probability that the server is in reserved

$$
\begin{aligned}
\mathrm{R} & =\mathrm{R}^{(1)}(1)+\mathrm{R}^{(2)}(1) \\
& =\mathrm{I}_{0} \lambda \mathrm{N}_{2}\left[\left(1-\mathrm{r}_{1}\right) \alpha_{1} \mu_{11} / \theta_{1}+(1-\tau)\left(1-\mathrm{r}_{2}\right) \alpha_{2} \mu_{21} / \theta_{2}\right] / \mathrm{T}_{1}
\end{aligned}
$$

Using the normalizing condition $\mathrm{I}_{0}+\mathrm{I}+\mathrm{P}+\mathrm{D}+\mathrm{F}+\mathrm{R}=1$ the expression of $\mathrm{I}_{0}$ is derived as $\mathrm{I}_{0}=\mathrm{T}_{1} / \mathrm{N}_{1}$ where

$\mathrm{T}_{1}=1-\mathrm{K}_{1}^{\prime}(1)-(1-\tau) \mathrm{K}_{2}^{\prime}(1)-\left(1-\mathrm{A}^{*}(\lambda)\right)\left(\mathrm{q}+\mathrm{m}_{1}-1\right)\left(\tau \rho_{1}+\rho_{2}(1-\tau)\right)$

$\mathrm{N}_{1}=\mathrm{A}^{*}(\lambda)+(1-\mathrm{q})\left(1-\mathrm{A}^{*}(\lambda)\right)\left(\tau \rho_{1}+\rho_{2}(1-\tau)\right)+\left(\mathrm{K}_{1}^{\prime}(1)\right.$ $\left.+(1-\tau) \mathrm{K}_{2}^{\prime}(1)\right)\left(\mathrm{A}^{*}(\lambda) \mathrm{m}_{1}(1-\mathrm{p})+(1-\mathrm{q})\left(1-\mathrm{A}^{*}(\lambda)\right)\right) /\left(\mathrm{pm}_{1}\right)$

$\mathrm{N}_{2}=\mathrm{m}_{1} \mathrm{~A} *(\lambda)+(1-\mathrm{q})\left(1-\mathrm{A}^{*}(\lambda)\right)$

$\mathrm{K}_{\mathrm{i}}^{\prime}(1)=\mathrm{p} \lambda \mu_{\mathrm{i} 1} \mathrm{~m}_{1}\left(1+\alpha_{\mathrm{i}}\left(\frac{1-\mathrm{r}_{\mathrm{i}}}{\theta_{\mathrm{i}}}+\mathrm{h}_{\mathrm{i} 1}+\mathrm{v}_{\mathrm{i} 1}\right)\right), \quad \mathrm{i}=1,2$.

The probability generating function of the number of customers in the retrial queue is given by

$$
\mathrm{P}_{\mathrm{q}}(\mathrm{z})=\mathrm{I}_{0}+\mathrm{I}(\mathrm{z})+\sum_{\mathrm{i}=1}^{2}\left[\mathrm{P}^{\mathrm{i}}(\mathrm{z})+\sum_{\mathrm{j}=0}^{1}\left[\mathrm{D}_{\mathrm{j}}^{\mathrm{i}}(\mathrm{z})+\mathrm{F}_{\mathrm{j}}^{\mathrm{i}}(\mathrm{z})\right]+\mathrm{R}^{\mathrm{i}}(\mathrm{z})\right]
$$

The probability generating function of the number of customers in the system is given by 
$\mathrm{P}_{\mathrm{s}}(\mathrm{z})=\mathrm{I}_{0}+\mathrm{I}(\mathrm{z})+\mathrm{z} \sum_{\mathrm{i}=1}^{2}\left[\mathrm{P}^{\mathrm{i}}(\mathrm{z})+\sum_{\mathrm{j}=0}^{1}\left[\mathrm{D}_{\mathrm{j}}^{\mathrm{i}}(\mathrm{z})+\mathrm{F}_{\mathrm{j}}^{\mathrm{i}}(\mathrm{z})\right]+\mathrm{R}^{\mathrm{i}}(\mathrm{z})\right]$

The mean number of customers in the orbit is

$$
\begin{aligned}
\mathrm{L}_{\mathrm{q}} & =\mathrm{P}_{\mathrm{q}}^{\prime}(1) \\
& =\frac{\mathrm{T}_{1} \mathrm{~N}_{4}+\mathrm{T}_{2} \mathrm{~N}_{3}+\mathrm{T}_{1} \mathrm{~N}_{3} \overline{\mathrm{C}}_{2} / 2 \overline{\mathrm{C}}_{1}}{\mathrm{p} \mathrm{T}_{1} \mathrm{~N}_{1} \overline{\mathrm{C}}_{1}}
\end{aligned}
$$

The mean number of customers in the system is

$$
\begin{aligned}
\mathrm{L}_{\mathrm{s}} & =\mathrm{P}_{\mathrm{s}}^{\prime}(1) \\
& =\frac{\mathrm{T}_{1} \mathrm{~N}_{5}+\mathrm{T}_{2} \mathrm{~N}_{3}+\mathrm{T}_{1} \mathrm{~N}_{3} \overline{\mathrm{C}}_{2} / 2 \overline{\mathrm{C}}_{1}}{\mathrm{p} \mathrm{T}_{1} \mathrm{~N}_{1} \overline{\mathrm{C}}_{1}}
\end{aligned}
$$

where

$$
\begin{aligned}
& \mathrm{T}_{2}= 1-\left(1-\mathrm{A}^{*}(\lambda)\right)\left(\tau \rho_{1}+\rho_{2}(1-\tau)\right)\left[\mathrm{K}_{1}^{\prime}(1)\left(\mathrm{q}+\mathrm{m}_{1}-1\right)\right. \\
&\left.+\mathrm{qm}_{1}+\mathrm{m}_{2} / 2\right]-\mathrm{K}_{1}^{\prime}(1)-(1-\tau) \mathrm{K}_{2}^{\prime}(1)\left[\mathrm{K}_{1}^{\prime}(1)\right. \\
&\left.+\left(1-\rho_{2}\right)+\rho_{2}\left(\mathrm{~A}^{*}(\lambda)+\left(1-\mathrm{A}^{*}(\lambda)\right)\left(\mathrm{q}+\mathrm{m}_{1}\right)\right)\right] \\
&-\left(\mathrm{K}_{1}^{\prime \prime}(1)+(1-\tau) \mathrm{K}_{2}^{\prime \prime}(1)\right) / 2 \\
& \mathrm{~N}_{3} \quad \mathrm{pm}_{1}\left(1-\mathrm{A}^{*}(\lambda)\right)\left[1-(1-\mathrm{q})\left(\tau \rho_{1}+\rho_{2}(1-\tau)\right)\right] \\
&-\left[\mathrm{K}_{1}^{\prime}(1)+(1-\tau) \mathrm{K}_{2}^{\prime}(1)\right]\left[\mathrm{m}_{1}(1-\mathrm{p}) \mathrm{A}^{*}(\lambda)\right. \\
&\left.+(1-\mathrm{q})\left(1-\mathrm{A}^{*}(\lambda)\right)\right] \\
& \mathrm{N}_{4}= \mathrm{p}\left[\tau \rho_{1}+\rho_{2}(1-\tau)\right]\left[( 1 - \mathrm { A } ^ { * } ( \lambda ) ) ( 1 - \mathrm { q } ) \left(\mathrm{m}_{1}\left(\mathrm{q}+\mathrm{K}_{1}^{\prime}(1)\right)\right.\right. \\
&\left.\left.+\mathrm{m}_{2} / 2\right)-\mathrm{m}_{1} \mathrm{~A}^{*}(\lambda) \mathrm{K}_{1}^{\prime}(1)\right]+\mathrm{p} \mathrm{A}^{*}(\lambda)\left[\mathrm{m}_{1}+\mathrm{m}_{2} / 2\right] \\
&+\mathrm{pm}_{1}(1-\tau)\left[\rho_{2}(1-\mathrm{q})\left(1-\mathrm{A}^{*}(\lambda)\right) \mathrm{K}_{2}^{\prime}(1)\right. \\
&\left.-\mathrm{K}_{2}^{\prime \prime}(1)\left(1-\rho_{2}\right) / 2\right] \\
&+\left[\mathrm{K}_{1}^{\prime}(1)+(1-\tau) \mathrm{K}_{2}^{\prime}(1)\right]\left[\mathrm{m}_{1}(1-\mathrm{q})\left(1-\mathrm{A}^{*}(\lambda)\right)\right. \\
&\left.+\mathrm{A}^{*}(\lambda)(1-\mathrm{p})\left(\mathrm{m}_{1}+\mathrm{m}_{2} / 2\right)\right]+\left[\mathrm{K}_{1}^{\prime \prime}(1)+(1-\tau) \mathrm{K}_{2}^{\prime \prime}(1)\right. \\
&\left.\left.+2(1-\tau) \mathrm{K}_{1}^{\prime \prime}(1) \mathrm{K}_{2}^{\prime \prime}(1)\right)\right]\left[\mathrm{N}_{2} \mathrm{pm}_{1} \mathrm{~A}^{*}(\lambda)\right] \\
&= \mathrm{N}_{4}+\mathrm{N}_{2}\left[\mathrm{~K}_{1}^{\prime \prime}(1)+(1-\tau) \mathrm{K}_{2}^{\prime \prime}(1)\right] \\
& \mathrm{N}_{5} \quad \\
& \mathrm{~K}_{\mathrm{i}}^{\prime \prime}(1)=\mathrm{p}^{2} \lambda^{2} \mathrm{~m}_{1}^{2}\left(\mu_{\mathrm{i} 2}\left(1+\alpha_{\mathrm{i}}\left(\frac{1-\mathrm{r}_{\mathrm{i}}}{\theta_{\mathrm{i}}}+\mathrm{h}_{\mathrm{i} 1}+\mathrm{v}_{\mathrm{i} 1}\right)\right)^{2}\right. \\
&\left.+2 \alpha_{\mathrm{i}} \mu_{\mathrm{i} 1}\left(\left(\frac{1-\mathrm{r}_{\mathrm{i}}}{\theta_{\mathrm{i}}}\right)\left(\frac{1}{\theta_{\mathrm{i}}}+\mathrm{h}_{\mathrm{i} 1}+\mathrm{v}_{\mathrm{i} 1}\right)+\left(\mathrm{h}_{\mathrm{i} 2}+2 \mathrm{~h}_{\mathrm{i} 1} \mathrm{v}_{\mathrm{i} 1}+\mathrm{v}_{\mathrm{i} 2}\right) / 2\right)\right) \\
&+\mathrm{p} \lambda \mathrm{m}_{2} \mu_{\mathrm{i} 1}\left(1+\alpha_{\mathrm{i}}\left(\frac{1-\mathrm{r}_{\mathrm{i}}}{\theta_{\mathrm{i}}}+\mathrm{h}_{\mathrm{i} 1}+\mathrm{v}_{\mathrm{i} 1}\right)\right), \mathrm{i}=1,2 \\
&
\end{aligned}
$$

\section{RELIABILITY INDEXES OF THE SERVER}

We now consider some reliability quantities of the server. Let $\mathrm{A}(\mathrm{t})$ be the system availability at time $\mathrm{t}$.

The steady state availability of the server A is

$$
\begin{aligned}
A= & \lim _{t \rightarrow \infty} A(t) . \\
= & \lim _{t \rightarrow \infty} P\{\text { the service station is up at time } t\} \\
= & I_{0}+\sum_{n=1}^{\infty} \int_{0}^{\infty} I_{n}(w) d w+\sum_{n=0}^{\infty} \sum_{i=1}^{2}\left[\int_{0}^{\infty} P_{n}^{i}(x) d x\right. \\
& \left.+\int_{0}^{\infty} \int_{0}^{\infty} R_{n}^{i}(x, r) d x d r\right] \\
= & \left\{A^{*}(\lambda)+\left(1-A^{*}(\lambda)\right)(1-q)\left(\tau \rho_{1}+\rho_{2}(1-\tau)\right)-A^{*}(\lambda) K_{1}^{\prime}(1)\right.
\end{aligned}
$$

$$
\begin{aligned}
& \left.+(1-\tau) \mathrm{K}_{2}^{\prime}(1)\right)+\lambda \mathrm{N}_{2}\left[\mu_{11}\left(1+\alpha_{1}\left(\frac{1-\mathrm{r}_{1}}{\theta_{1}}\right)\right)\right. \\
& \left.\left.+(1-\tau) \mu_{21}\left(1+\alpha_{2}\left(\frac{1-\mathrm{r}_{2}}{\theta_{2}}\right)\right)\right]\right\} / \mathrm{N}_{1}
\end{aligned}
$$

The steady state failure frequency of the server is

$$
\begin{aligned}
\mathrm{F} & =\sum_{\mathrm{n}=0}^{\infty} \sum_{\mathrm{i}=1}^{2} \int_{0}^{\infty} \alpha_{\mathrm{i}} \mathrm{P}_{\mathrm{n}}^{\mathrm{i}}(\mathrm{x}) \mathrm{dx} \\
& =\mathrm{N}_{2}\left[\alpha_{1} \mu_{11}+\alpha_{2}(1-\tau) \mu_{21}\right] / \mathrm{N}_{1}
\end{aligned}
$$

\section{SPECIAL CASES}

The model under study is a generalization of many queueing models.

Case 1: If $\mathrm{C}(\mathrm{z})=\mathrm{z}$, then the model reduces to $\mathrm{M} / \mathrm{G} / 1$ two phase retrial queueing system with impatient customers,server breakdown, delayed repair and orbital search.

Case 2: If $\mathrm{C}(\mathrm{z})=\mathrm{z}$ and $\rho_{1}=\rho_{2}=1$ the system reduces to two phase retrial queueing system with impatient customers, server breakdown, delayed repair and reserved time [9].

Case 3: If $C(z)=z$ and $p=q=\rho_{1}=\rho_{2}=1$ the system reduces to two phase retrial queueing system with server breakdown, delayed repair and reserved time [16].

Case 4: If $\tau=1$ and $p=q=\rho_{1}=\rho_{2}=1$ the system reduces to impatient customer bulk arrival retrial queueing system with server breakdown and delayed repair [12].

Case 5: If $\tau=1=r_{1}=r_{2}=\rho_{1}=\rho_{2}=1$ the system coincides with the paper bulk arrival retrial queueing system with server breakdown and delayed repair [13].

\section{NUMERICAL RESULTS}

Assume that the distributions of service time, retrial time, delay time and repair time follow (i) Erlangian of order 2, (ii) Exponential and (iii) Hyper-exponential distributions. Table 1 provides the availability of the server $A$ and the failure frequency F by varying the values of $\mu_{1}, \tau$ and $\alpha_{1}$ for the three processes Erlang, exponential and hyper-exponential with the fixed values $\left(\lambda, \eta, \mu_{2}, \gamma_{1}, \gamma_{2}, \beta_{1}, \beta_{2}, r_{1}, r_{2}, \rho_{1}, \rho_{2}, \theta_{1}, \theta_{2}, C_{1}, C_{2}, \alpha_{2}, p, q, a\right)=(1$, $15,20,5,6,3,6,0.5,0.5,0.5,0.5,6,3,0.6,0.4,2,0.8,0.8,0.5$ ).

For all $\mu_{1}$ and $\tau$, as $\alpha_{1}$ increases the availability of the server decreases and the failure frequency increases. This can be explained intuitively as follows: As failure rate $\alpha_{1}$ increases life time decreases and hence availability decreases and failure rate increases. 
Table 1. Availability and Failure frequency

\begin{tabular}{|c|c|c|c|c|c|c|c|c|}
\hline \multirow{2}{*}{$\mu_{1}$} & \multirow{2}{*}{$\tau$} & \multirow{2}{*}{$\alpha_{1}$} & \multicolumn{2}{|l|}{ Erlang } & \multicolumn{2}{|c|}{ Exponential } & \multicolumn{2}{|c|}{ Hyper-exponential } \\
\hline & & & A & $\mathrm{F}$ & A & $\mathrm{F}$ & A & $\mathrm{F}$ \\
\hline \multirow{15}{*}{25} & \multirow{5}{*}{0.0} & 1 & 0.7282 & 0.3480 & 0.9268 & 0.1876 & 0.9762 & 0.1006 \\
\hline & & 2 & 0.6313 & 0.4366 & 0.8989 & 0.2395 & 0.9665 & 0.1289 \\
\hline & & 3 & 0.5389 & 0.5211 & 0.8714 & 0.2907 & 0.6569 & 0.1569 \\
\hline & & 4 & 0.4507 & 0.6018 & 0.8442 & 0.3413 & 0.9473 & 0.1849 \\
\hline & & 5 & 0.3665 & 0.6780 & 0.8175 & 0.3912 & 0.9377 & 0.2127 \\
\hline & \multirow{5}{*}{0.5} & 1 & 0.8041 & 0.2320 & 0.9484 & 0.1223 & 0.9831 & 0.0650 \\
\hline & & 2 & 0.7017 & 0.3267 & 0.9199 & 0.1754 & 0.9733 & 0.0935 \\
\hline & & 3 & 0.6044 & 0.4169 & 0.8919 & 0.2278 & 0.9636 & 0.1219 \\
\hline & & 4 & 0.5116 & 0.5028 & 0.8643 & 0.2795 & 0.9540 & 0.1501 \\
\hline & & 5 & 0.4231 & 0.5846 & 0.8370 & 0.3305 & 0.9443 & 0.1782 \\
\hline & \multirow{5}{*}{1} & 1 & 0.8858 & 0.1071 & 0.9706 & 0.0551 & 0.9901 & 0.0289 \\
\hline & & 2 & 0.7775 & 0.2086 & 0.9416 & 0.1095 & 0.9803 & 0.0577 \\
\hline & & 3 & 0.6747 & 0.3050 & 0.9130 & 0.1631 & 0.9705 & 0.0863 \\
\hline & & 4 & 0.5769 & 0.3967 & 0.8848 & 0.2159 & 0.9607 & 0.1148 \\
\hline & & 5 & 0.4838 & 0.4840 & 0.8570 & 0.2680 & 0.9510 & 0.1432 \\
\hline \multirow{15}{*}{30} & \multirow{5}{*}{0.0} & 1 & 0.7440 & 0.3340 & 0.9313 & 0.1792 & 0.9779 & 0.0958 \\
\hline & & 2 & 0.6619 & 0.4090 & 0.9080 & 0.2227 & 0.9698 & 0.1193 \\
\hline & & 3 & 0.5831 & 0.4810 & 0.8849 & 0.2657 & 0.9618 & 0.1426 \\
\hline & & 4 & 0.5074 & 0.5503 & 0.8620 & 0.3082 & 0.9538 & 0.1658 \\
\hline & & 5 & 0.4345 & 0.6169 & 0.8394 & 0.3503 & 0.9459 & 0.1890 \\
\hline & \multirow{5}{*}{0.5} & 1 & 0.8210 & 0.2165 & 0.9531 & 0.1136 & 0.9484 & 0.0601 \\
\hline & & 2 & 0.7343 & 0.2968 & 0.9292 & 0.1581 & 0.9767 & 0.0838 \\
\hline & & 3 & 0.6512 & 0.3737 & 0.9057 & 0.2021 & 0.9686 & 0.1073 \\
\hline & & 4 & 0.5715 & 0.4475 & 0.8824 & 0.2455 & 0.9606 & 0.1308 \\
\hline & & 5 & 0.4948 & 0.5185 & 0.8594 & 0.2885 & 0.9526 & 0.1542 \\
\hline & \multirow{5}{*}{1} & 1 & 0.9040 & 0.0900 & 0.9754 & 0.0461 & 0.9918 & 0.0240 \\
\hline & & 2 & 0.8123 & 0.1760 & 0.6511 & 0.0916 & 0.9836 & 0.0479 \\
\hline & & 3 & 0.7244 & 0.2584 & 0.6271 & 0.1366 & 0.9755 & 0.0716 \\
\hline & & 4 & 0.6402 & 0.3373 & 0.6034 & 0.1811 & 0.9674 & 0.0953 \\
\hline & & 5 & 0.5595 & 0.4130 & 0.8800 & 0.2251 & 0.9593 & 0.1189 \\
\hline \multirow{15}{*}{35} & \multirow{5}{*}{0.0} & 1 & \begin{tabular}{|l|l|}
0.7553 \\
\end{tabular} & \begin{tabular}{|l|}
0.32338 \\
\end{tabular} & 0.9346 & 0.1731 & 0.9790 & 0.0925 \\
\hline & & 2 & 0.6842 & 0.3889 & 0.9145 & 0.2106 & 0.9722 & 0.1125 \\
\hline & & 3 & 0.6155 & 0.4516 & 0.8946 & 0.2476 & 0.9653 & 0.1324 \\
\hline & & 4 & 0.5492 & 0.5123 & 0.8749 & 0.2844 & 0.9585 & 0.1523 \\
\hline & & 5 & 0.4850 & 0.5710 & 0.8554 & 0.3207 & 0.9517 & 0.1721 \\
\hline & \multirow{5}{*}{0.5} & $\begin{array}{lll} & \end{array}$ & 0.8333 & 0.2053 & 0.9564 & 0.1073 & 0.9860 & 0.0567 \\
\hline & & 2 & 0.7581 & 0.2749 & 0.9359 & 0.1456 & 0.9790 & 0.0769 \\
\hline & & 3 & 0.6856 & 0.3420 & 0.9156 & 0.1835 & 0.9721 & 0.0971 \\
\hline & & 4 & 0.3157 & 0.4067 & 0.8956 & 0.2211 & 0.9653 & 0.1171 \\
\hline & & 5 & 0.5481 & 0.4693 & 0.8757 & 0.2582 & 0.9584 & 0.1371 \\
\hline & \multirow{5}{*}{1} & 1 & 0.9173 & 0.0776 & 0.9789 & 0.0396 & 0.9930 & 0.0205 \\
\hline & & 2 & 0.8376 & 0.1522 & 0.9580 & 0.0788 & 0.9860 & 0.0409 \\
\hline & & 3 & 0.7610 & 0.2241 & 0.9373 & 0.1176 & 0.9790 & 0.0612 \\
\hline & & 4 & 0.6841 & 0.2934 & 0.9168 & 0.1560 & 0.9721 & 0.0815 \\
\hline & & 5 & 0.6158 & 0.3602 & 0.8965 & 0.1940 & 0.9652 & 0.1017 \\
\hline
\end{tabular}

\section{REFERENCES}

[1] Aissani, A. (2010), "An M/G/1 Retrial Queue with Negative Arrivals and Unreliable Server", Proceeding of the World Congress On Engineering, Vol. I, June 30 - July 2, London, UK.

[2] Artalejo, J. R. and Gomez-Corral, A. (2008), "Retrial Queueing Systems - A Computational Approach", Springer Verlag, Berlin.

[3] Artalejo, J.R. (1999), "A classified bibliography of research on retrial queues : Progress in 1990, 1999", Top., 7, 187211.

[4] Artalejo, J.R. (1999), "Accessible bibliography on retrial queues", Mathematical and Computer Modelling, 30, 1-6.

[5] Artalejo, J.R., Joshua, V.C. and Krishnamoorthy, A. (2002), "An M/G/1 retrial queue with orbital search by the server", In Advances in Stochastic Modelling, eds, Artalejo, J.R. and Krishnamoorthy, A., 41-54, New Jersey : Notable Publications.
[6] Atecia, I., Bouza,G. and Moreno, P. (2008), "An M ${ }^{[x]} / \mathrm{G} / 1$ retrial Queue with server breakdowns and constant rate of repeated attempts", Ann. Oper. Res., 157: 225-243.

[7] Chakravarthy, S.R., Krishnamoorthy, A. and Joshua, V.C (2006), "Analysis of a multiserver retrial queue with search of customers from the orbit", Performance Evaluation, 63, 8, $776-798$.

[8] Dudin, A.N., Krishnamoorthy, A., Joshua, V.C. and Tsarenkov, G. (2004), "Analysis of the BMAP/G/1 retrial system with search of customers from the orbit", European Journal of Operational Research, 157, 169-179.

[9] Ebenesar Annabagyam, J. and Udaya Chandrika, K. (2012), "Two Phase Retrial Queueing System with Impatient Customers, Additional Optional Service, Server Breakdown, Delayed Repair and Reserved Time", International Journal of Mathematical Archive, 3, 2283 2290 .

[10] Falin, G. I. and Templeton, J. G. C. (1997), "Retrial Queues", Chapman and Hall, London.

[11] Geni Gupur (2010) , "Analysis of the M/G/1 retrial queueing model with server breakdowns", J. Pseudo-Differ. Oper. Appl.,1:313-340.

[12] Prakash Rani, K., Sinivasan, A. and Udaya Chandrika, K. (2009), "Impatient customers Bulk Arrival Retrial Queue with Server Breakdown and Delayed Repair", Advances In Applied Research, 1, 1, 60-68.

[13] Prakash Rani, K., Sinivasan, A. and Udaya Chandrika, K. (2011), "Retrial queue with Server Breakdown and Delayed Repairs", Proceedings of the International Congress on Productivity,Quality Reliability, Optimization and Modelling(ICPQROM 2011), 34-47, ISBN 978-81-842-7084, Allied Publishers Pvt.Ltd

[14] Rehab F. Khalaf, Kailash C. Madan and Cormac A. Lukas (2011), “ An $\mathrm{M}^{[\mathrm{x}]} / \mathrm{G} / 1$ Queue with Bernoulli Schedule, General Vacation Times, Random Breakdowns, General Delay Times and General Repair Times", Applied Mathematical Sciences, Vol. 5, No.1 , 25-51.

[15] Udaya Chandrika, K. and Ebenesar Annabagyam, J. (2008), "Two Phase Retrial Queue With impatient customers and Orbital search", Research Highlights, 18, 3, 152 - 157.

[16] Udaya Chandrika, K. and Ebenesar Annabagyam, J. (2011), "Two phase retrial queueing system with active breakdown deayed repair and reserved time", Proceedings of the National Conference on Recent Advancements in Science and Humanities, 54, ISBN: 978-81-920799-1-2.

[17] Wang, J. (2004), "An M/G/1 Queue with Second Optional Service and Server Breakdowns", Computers and Mathematics with Applications, 47: 1713-1723.

[18] Wang, J. and Li H. (1994), "Reliability Analysis of the retrial Queue with server breakdowns and repairs", Queueing Systems, 38, 363-380. 